\title{
Break-Even Inflation Rate and the Risk Premium: An Alternative Approach to the VAR Models in Forecasting the CPI
}

\author{
João F. Caldeira ${ }^{1} \quad$ Luiz G. C. Furlani $^{2}$
}

Maio 2011

\begin{abstract}
This paper examines, for the Brazilian case, if break-even inflation rates (BEIR) extracted from fixed income securities is an unbiased estimator of consumer inflation, measured by the CPI. Our estimates suggest that BEIRs are informative about future inflation, especially for the maturity of three months. The main innovation of our work, however, is the method used for estimation, allowing us to conclude that the inflation risk premium, for some maturities considered, varies over time and is not irrelevant from the economic standpoint. We also compared the inflation forecasts obtained from BEIRs with the ones extracted from VAR models used by Central Bank and estimates from the Focus Survey Report's Top5s. The forecasts performed with BEIRs showed greater accuracy than those extracted from VAR models. These projections, however, underperformed those from the Top5s.
\end{abstract}

JEL Classification: E31, E43, E44.

Keywords: break-even inflation rate, inflation expectations, inflation.

\footnotetext{
${ }^{1}$ Department of Economics and PPGE-UFRGS. E-mail: joao.caldeira@.ufrgs.br.

${ }^{2}$ PPGE-UFRGS and SICREDI. E-mail: luiz_furlani@sicredi.com.br.
} 


\section{Introduction}

The anchoring of inflation expectations is critical to the conduct of monetary policy and price stability in the economy, especially for a Central Bank that follows an inflationtargeting regime. For this reason, monetary authorities around the world seek relentlessly reliable and appropriate indicators to monitor inflation expectations. Inflation-linked bonds are one of the most important real-time monitoring sources of future inflation expectations, according to officials from the European Central Bank and the Federal Reserve System, including current chairman of the Board of Governors, Ben Bernanke.

The use of the above mentioned bonds can be easily justified: given their forwardlooking characteristic, besides a risk premium, they incorporate investors' expectations about future inflation. Many studies investigated and supported the use of inflation-linked bonds to monitor inflation expectations. Among others, we can cite Hetzel (1992), Breedon (1995), Campbell and Shiller (1996), Deacon and Andrews (1996), Barr and Campbell (1997), Kitamura (1997), Evans (1998), Emmons (2000), Jarrow and Yildirim (2003), Bernanke (2004), Buraschi and Jiltsov (2005), Hordahl et al. (2005), Liu and Cheng (2005), D’Amico

et al. (2006), Ang et al. (2007), Garcia and van Rixtel (2007), Haubrich et al. (2008) and Grishenko and Huang (2010).

For Brazil, Vincent and Guillen (2010) analyze whether inflation-linked bonds contain information about future inflation. To do so, they consider a series of regressions between realized inflation, break-even inflation rate (BEIR) - i.e., the spread between real and nominal yields -, as well as a constant term. They find out that BEIR is informative and an unbiased estimator for inflation at 3 and 6 months horizons. The BEIR is also informative when considered maturities of 24 and 30 months, however, has no explanatory power for 12 and 18 months. According to the authors, the lack of explanatory power for intermediate 
horizons can be explained, among other factors, by the higher reward required by investors to hold those bonds.

This reward - i.e., risk premium -, according to Garcia and van Rixtel (2007), is basically due to two factors: the first is associated to a compensation for uncertainty about future inflation, especially in bonds with longer maturities, while the second refers to a compensation for liquidity. This means that there is a compensation for the incurred risk and that this compensation, not necessarily, is constant over time, as assumed and Vicente and Guillen (2010).

Without those restrictive assumptions imposed by Vicente and Guillen (2010), the present paper examines, for the Brazilian case, if break-even inflation rates extracted from fixed income securities is an unbiased estimator of consumer inflation rates. To accomplish this primary objective, we allow the risk premium and the explanatory power of the BEIR to vary over time. This paper also evaluates the predictive power of the BEIR against other measures of inflation expectations, such as those extracted from VAR models used by the Central Bank of Brazil and estimates from the Focus Survey Report's Top5s.

The remainder of this paper is organized as follows: section 2 presents the econometric model used to meet the main objective of this study; section 3 offers details about the data used and the treatment given to them; section 4 presents the estimation results; in section 5, we evaluate the predictive power of the BEIR against the inflation expectations extracted from the VAR models and the Focus Survey Report; and, finally, section 6 concludes.

\section{The Model}

The model we use to assess whether break-even inflation rates are informative about future inflation is similar to the one proposed by Vicente and Guillen (2010). Let $y_{t}^{N}(\tau)$ and 
$y_{t}^{R}(\tau)$ be, respectively, continuously compounded real and nominal yields at time $t$ to maturity $\tau$. The BEIR is defined as the spread between nominal and real yields, given by:

$$
i_{t}(\tau)=y_{t}^{N}(\tau)-y_{t}^{R}(\tau)
$$

The annual rate of change in consumer prices between two periods - i.e., the inflation rate observed in that period,$- t$ and $t+\tau$, for example, is given by $h_{t}(\tau)$ :

$$
h_{t}(\tau)=\frac{1}{\tau} \sum_{j=t}^{t+\tau-1} h_{j}(\tau)
$$

The information content of the BEIR can be assessed, according to Vicente and Guillen (2010), by estimating the following equation:

$$
h_{t}(\tau)=c_{1}^{\tau}+c_{2}^{\tau} i_{t}(\tau)+\varepsilon_{t}
$$

If $c_{2}^{\tau}$ is nonzero, the BEIR contains information about future inflation. Furthermore, if $c_{2}^{\tau}$ is equal to one and $c_{1}^{\tau}$ is zero, the BEIR is an unbiased estimator of the future inflation. Intuitively, it is easy to verify this relationship. To do so, we simply rewrite the equation (3) for future inflation: the spread between nominal and real yields will be equal to the expected future inflation plus a compensation for the incurred risk.

However, as mentioned earlier, we wish to estimate (3) without imposing any restrictions on the risk premium, since there is evidence that it might not be constant over time, as suggested by Grishchenko and Huang (2010). The coefficient associated with the 
BEIR is not necessarily constant over time as well and, thus, we also allow it to be timevarying. To do so, we have to estimate the following system:

$$
\begin{aligned}
& h_{t}(\tau)=c_{1, t}^{\tau}+c_{2, t}^{\tau} i_{t}(\tau)+\varepsilon_{t} \\
& c_{1, t}^{\tau}=c_{1, t-1}^{\tau}+\eta_{t} \\
& c_{2, t}^{\tau}=c_{2, t-1}^{\tau}+\zeta_{t}
\end{aligned}
$$

The system described in (4) will be estimated by the Kalman Filter, so it must be rewritten in a state space representation. The measurement equation (5), relates the vector of observations with the state vector, the explanatory variable and the measurement error, while the transition equation (6) describes the dynamics of the state variables, in the form of a firstorder difference equation in the state vector:

$$
\begin{aligned}
& {\left[h_{t}(\tau)\right]=\left[\begin{array}{ll}
c_{1, t}^{\tau} & c_{2, t}^{\tau}
\end{array}\right]\left[\begin{array}{c}
1 \\
i_{t}(\tau)
\end{array}\right]+\left[\varepsilon_{t}\right]} \\
& {\left[\begin{array}{l}
C_{1, t}^{\tau} \\
c_{2, t}^{\tau}
\end{array}\right]=\left[\begin{array}{ll}
1 & 0 \\
0 & 1
\end{array}\right]\left[\begin{array}{c}
C_{1, t-1}^{\tau} \\
C_{2, t-1}^{\tau}
\end{array}\right]+\left[\begin{array}{c}
\eta_{t} \\
\zeta_{t}
\end{array}\right]}
\end{aligned}
$$

Concisely, the objective of the Kalman filter is to recursively obtain the conditional distribution of the states, given the model and the data. Considering that the variances of the innovations are not directly observable, the procedure starts in its estimation. First, through a numerical procedure that guarantees positivity, the variances of the innovations are estimated by maximum likelihood. In addition to the variances of innovations, we also get the vector of filtered states and its covariance matrix. To obtain the smoothed state vector and the associated covariance matrix, we apply the Kalman filter backwards, finishing the estimation procedure. 


\section{The Data}

The database we used consists of monthly series of nominal and real yields for the period of January 2005 to January 2010, extracted from the ANBIMA website - the Brazilian Association of Investment Banks and Financial Market Institutions. The term structure of nominal interest rates is extracted from the yields of NTN-Fs and LTNs, issued by the National Treasury. The face value of an NTN-F is R $\$ 1,000.00$, with a semiannual coupon payment of $\mathrm{R} \$ 48.81$. The LTN is a zero-coupon bond with face value of $\mathrm{R} \$ 1,000.00$. We use the model of Svensson (1994) to adjust the yield curve for the desired maturities.

The term structure of real interest rates is also built using the model of Svensson (1994). However, the curve is adjusted using NTN-Bs - the main class of inflation-linked bonds issued by the National Treasury. The yield of an NTN-B is determined by the IPCA, the consumer price index adopted in the inflation-targeting regime by the Central Bank of Brazil. It is worthy noticing that NTN-Bs do not have the problem of indexation lag, as in the TIPS market, since interest is paid based on the current level of the IPCA, available with a lag not greater than 15 days. Although the NTN-Bs are issued since 2001, we began our database in January 2005, to avoid liquidity problems.

The estimation of the term structure of zero-coupon inflation-indexed bonds and corresponding BEIRs to Brazil present two main advantages in comparison with the measures discussed earlier in this article. First, it allows the estimation of a time series of real interest rates and BEIRs with fixed maturities, which is particularly useful when analyzing their evolution over a relatively long period of time. And second, the calculation of equivalent zero-coupon yields allows us to avoid potential distortions related to different durations in the securities used to compute the BEIRs. Such distortions are related to differences in the cash flow structures of inflation-linked bonds and nominal bonds. 
Nonetheless, the estimation of term structures for Brazil requires the solution of some technical problems related to the small number of available inflation-indexed bonds, particularly for shorter maturities. Despite the significant market growth of inflation-linked bonds in recent years, there are still some limitations in this regard. As mentioned previously, to estimate comparable term structures of nominal and real yields to the Brazilian market, we use the parametric approach proposed by Svensson (1994). This method assumes that the zero-coupon yield to maturity $\tau, y(\tau)$, is specified by the following functional form:

$$
y(\tau)=\beta_{1}+\beta_{2}\left(\frac{1-e^{\lambda_{1} \tau}}{e^{\lambda_{1} \tau}}\right)+\beta_{3}\left(\frac{1-e^{\lambda_{1} \tau}}{e^{\lambda_{1} \tau}}-e^{\lambda_{1} \tau}\right)+\beta_{4}\left(\frac{1-e^{\lambda_{2} \tau}}{e^{\lambda_{2} \tau}}-e^{\lambda_{2} \tau}\right)
$$

The parameters $\beta_{1}, \beta_{2}, \beta_{3}, \beta_{4}$ and $\lambda$ can be estimated by minimizing the difference between bond prices implied by the functional form and observed bond prices. This methodology is applied to the estimation of comparable nominal and real yield curves, generating BEIRs with fixed maturities.

The Brazilian market for inflation-linked bonds is one of the largest worldwide, with over US\$ 200 billion in NTN-Bs in circulation. The average maturity of NTN-Bs is approximately six years. The Brazilian market for fixed income securities is also significant. The LTNs and NTN-Fs have around US\$ 155 billion and US\$ 126 billion in circulation, with average maturities of 12 months and 30 months respectively. 
Table 1 - Descriptive Statistics

\begin{tabular}{|c|c|c|c|c|c|c|c|c|c|}
\hline Maturities(Months) & Mean & $\begin{array}{l}\text { Standard } \\
\text { Deviation }\end{array}$ & Max. & Min. & Asymmetry & Kurtosis & Jarque-Bera & Correlation & ACF1 \\
\hline 3 & $4.96 \%$ & $1.25 \%$ & $10.39 \%$ & $3.20 \%$ & 2.07 & 3.31 & 5.13 & 0.32 & 0.67 \\
\hline 6 & $5.06 \%$ & $1.64 \%$ & $11.67 \%$ & $2.68 \%$ & 2.17 & 5.23 & 23.21 & 0.30 & 0.76 \\
\hline 9 & $4.92 \%$ & $1.54 \%$ & $10.92 \%$ & $2.93 \%$ & 2.06 & 4.81 & 22.49 & 0.35 & 0.78 \\
\hline 12 & $4.80 \%$ & $1.38 \%$ & $10.12 \%$ & $3.20 \%$ & 2.03 & 5.17 & 32.58 & 0.36 & 0.79 \\
\hline 15 & $4.75 \%$ & $1.26 \%$ & $9.48 \%$ & $3.23 \%$ & 1.92 & 5.37 & 30.05 & 0.37 & 0.79 \\
\hline 18 & $4.76 \%$ & $1.19 \%$ & $9.01 \%$ & $3.22 \%$ & 1.77 & 5.27 & 27.52 & 0.37 & 0.80 \\
\hline 24 & $4.87 \%$ & $1.12 \%$ & $8.38 \%$ & $3.25 \%$ & 1.38 & 4.42 & 20.31 & 0.36 & 0.80 \\
\hline 30 & $4.95 \%$ & $1.14 \%$ & $8.00 \%$ & $3.32 \%$ & 1.12 & 3.90 & 9.72 & 0.35 & 0.84 \\
\hline 36 & $5.00 \%$ & $1.20 \%$ & $7.78 \%$ & $3.39 \%$ & 1.00 & 3.73 & 6.37 & 0.31 & 0.85 \\
\hline CPI & $4.89 \%$ & $2.77 \%$ & $10.95 \%$ & $-2.49 \%$ & 0.01 & 2.97 & 0.04 & 1.00 & 0.55 \\
\hline Level & $6.83 \%$ & $1.17 \%$ & $9.76 \%$ & $5.50 \%$ & 1.20 & 4.39 & 17.11 & $\begin{array}{l}-0.04 \\
\end{array}$ & 0.90 \\
\hline Slope & $0.17 \%$ & $3.27 \%$ & $9.69 \%$ & $-6.82 \%$ & 0.10 & 3.16 & 0.45 & 0.12 & 0.43 \\
\hline
\end{tabular}

Table 1 presents some descriptive statistics of the BEIRs and the IPCA - henceforth, for simplicity, CPI. The average CPI for all horizons is about 4.9\%. Both BEIRs and inflation are leptokurtic, with positive asymmetry, i.e., long tails on the right. The Jarque-Bera statistic indicates that BEIR for 3 months horizon and the CPI appear to be normally distributed. This is an indication of the predictive power of the BEIR for short horizons. This evidence is confirmed in the empirical exercise conducted in section 4. The correlation coefficients between realized inflation and BEIRs are positive for all analyzed horizons.

The autocorrelation coefficients with one-month lag indicate high persistence of BEIRs for all maturities, with all the autoregressive coefficients above 0.65 . In the last two lines of table 1, we present the descriptive statistics of the level and slope of the estimated term structure for the BEIRs. The average level is $6.83 \%$, showing high persistence. The slope factor has positive average, with autoregressive coefficient of 0.43 .

Figure 1 depicts the evolution of the BEIRs and inflation over time, for the period of January 2005 to January 2011. It is important to note that the term structure of the BEIRs is almost always positively sloped. However, the BEIRs and the CPI do not exhibit any trend. 
Figure 1 - Evolution of the BEIRs and Inflation

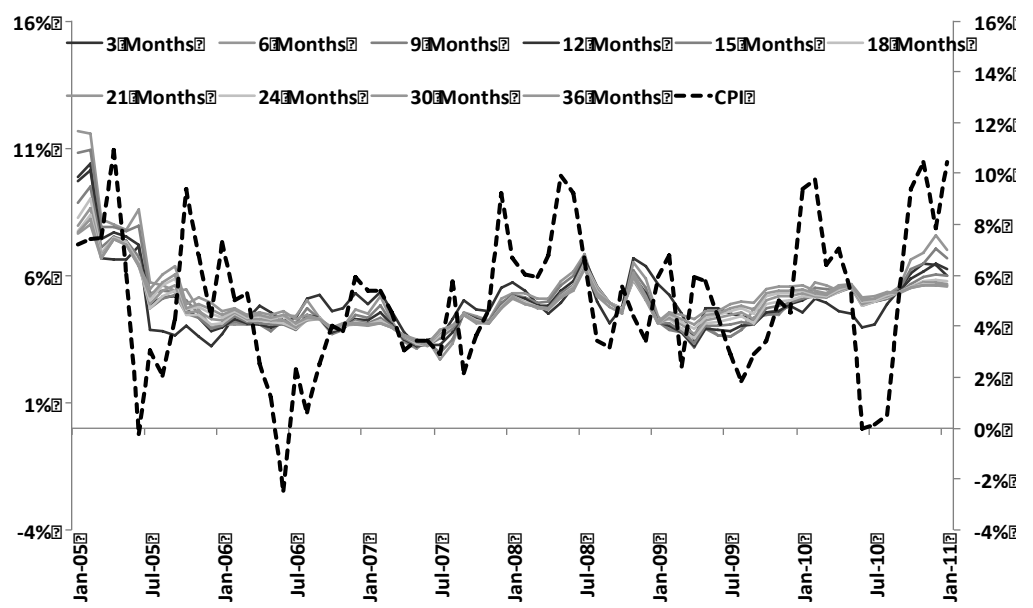

\section{Estimation and Results}

After estimating the comparable nominal and real yield curves with fixed maturities, using the model of Svensson (1994) and methodology described in the previous section, the BEIRs were computed and the system represented by the equations in (4) estimated. The system has been estimated by the Kalman filter to allow its coefficients to vary over time and, thus, not impose any restrictions on the risk premium and the coefficient associated with the BEIRs. As a robustness test of the results, we have also estimated a version of the system in equations (4) without time-varying coefficients, given by equation (3), by ordinary least squares.

Table 2 - Kalman Filter and Ordinary Least Squares Estimates

\begin{tabular}{|c|c|c|c|c|c|c|c|c|c|}
\hline \multicolumn{10}{|c|}{ State Space - Kalman Filter } \\
\hline & 3 & 6 & 9 & 12 & 15 & 18 & 24 & 30 & 36 \\
\hline c1 & $0.0341 * *$ & $0.0380 * *$ & $0.0414 * *$ & $0.0363 * *$ & $0.0546 * *$ & $0.0522 * *$ & $0.0532 * *$ & $0.0519 * *$ & $0.0535^{* *}$ \\
\hline c2 & $0.6331 * *$ & $0.2002 * *$ & $0.1561^{* *}$ & 0.2665 & 0.0344 & $-0.0161 * *$ & $-0.0468 * *$ & $-0.0396 * *$ & $-0.0568 * *$ \\
\hline \multicolumn{10}{|c|}{ Ordinary Least Squares } \\
\hline & 3 & 6 & 9 & 12 & 15 & 18 & 24 & 30 & 36 \\
\hline c1 & $0.0203 * *$ & $0.0356 * *$ & $0.0384 * *$ & $0.0402 * *$ & $0.0465 * *$ & $0.0531 * *$ & $0.0590 * *$ & $0.0616 * *$ & $0.0584 * *$ \\
\hline c2 & $0.5781^{* * *}$ & $0.2311^{*}$ & $0.1792 *$ & 0.1462 & 0.0073 & -0.1397 & $-0.2444 * *$ & $-0.2813 * *$ & $-0.2042 * *$ \\
\hline
\end{tabular}


Table 2 shows the estimated coefficients by the Kalman filter, in the smoothed final state, and by ordinary least squares. Overall, the results are similar for all maturities, both in magnitude of the estimated coefficients, as in statistical significance of these coefficients. Considering the results obtained by the estimation of state space representation, the coefficients associated with the BEIRs proved to be positive and statistically significant for 3, 6 and 9 months. This means that, for these maturities, BEIR brings relevant information about future inflation.

For 12 and 15 months, the coefficients associated with the BEIR are not statistically significant, while for $18,24,30$ and 36 months, the coefficients, though significant, are negative. This negative relationship for longer maturities, according to Vincent and Guillen (2010), implies that the expectations hypothesis does not apply in the long term and, thus, equation (3) may not be adequate to explain the relationship between inflation and BEIR. One possible solution to this problem, according to the authors, would be to relax the assumption of constant risk premium. The relaxation of this hypothesis has been considered in the system described in (4), estimated by the Kalman filter, and yet, the coefficients associated with the BEIRs remained negative for 18, 24, 30 and 36 months. This means that the relationship between inflation and BEIR can be highly complex.

The constant term, representing the compensation for the incurred risk, is statistically different from zero - though very close to that value - for all maturities. It is noteworthy, however, that, when estimated by the Kalman filter, the system in (4) generated time-varying risk premium for some maturities, such as $15,18,24,30$ and 36 months. 
Figure 2 - Risk Premium, Smoothed States
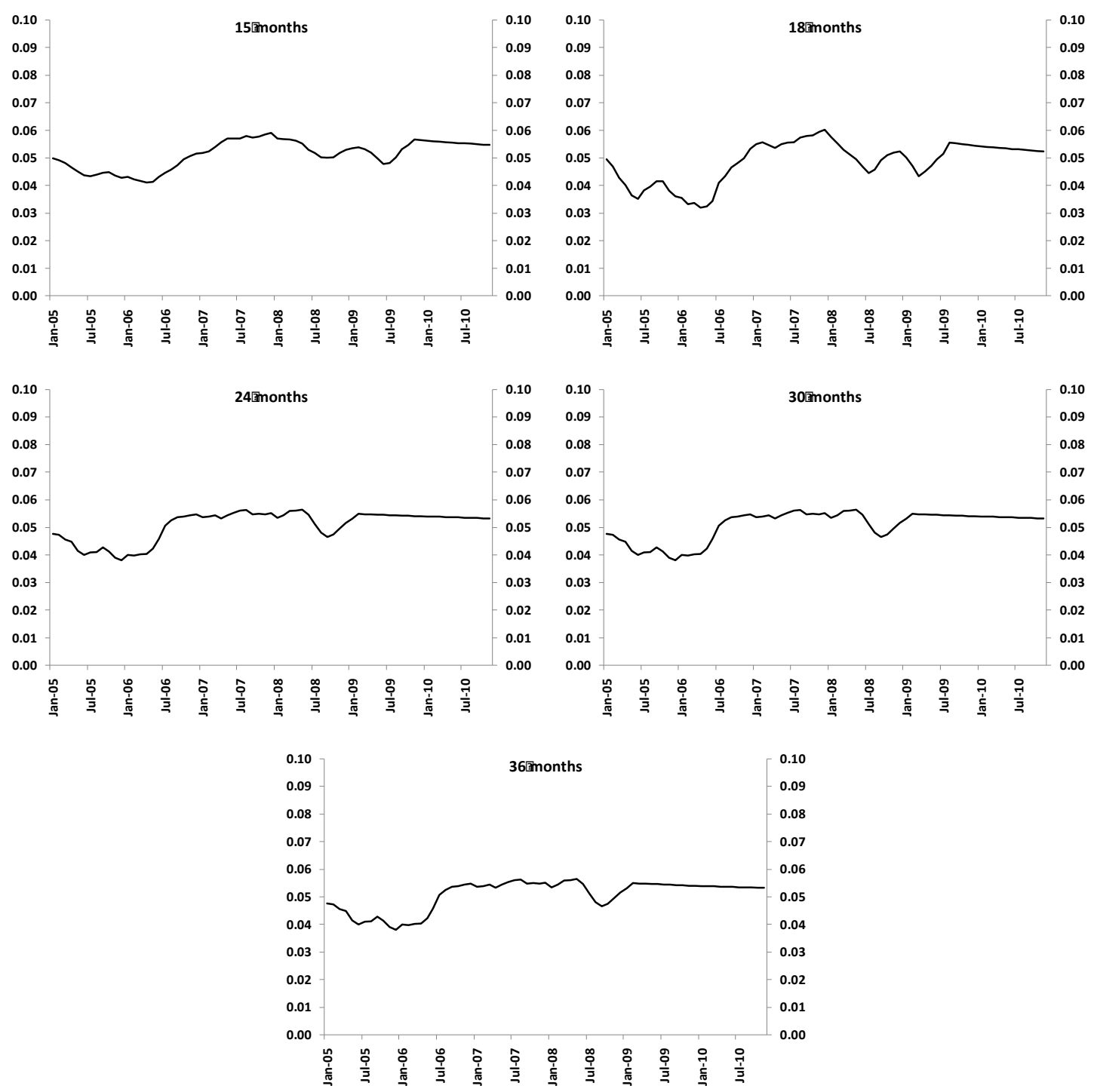

Since, for almost all maturities, the BEIR carries information about future inflation, it becomes interesting to assess whether it can be considered an unbiased estimator for inflation. For this to be true, it is necessary that, jointly, $c_{1}^{\tau}=0$ and $c_{2}^{\tau}=1$. We performed, then, a F-test to assess the validity of this hypothesis for each of the maturities considered. 
Table 3 - F-Statistics for $c_{1}^{\tau}=0$ and $c_{2}^{\tau}=1$

\begin{tabular}{|c|c|c|c|c|c|c|c|c|c|}
\hline \multicolumn{10}{|c|}{ F-Statistics } \\
\hline & 3 & 6 & 9 & 12 & 15 & 18 & 24 & 30 & 36 \\
\hline $\begin{array}{l}c 1=0 \\
c 2=1\end{array}$ & $0.1230 * *$ & 0.0000 & 0.0000 & 0.0000 & 0.0000 & 0.0000 & 0.0000 & 0.0000 & 0.0000 \\
\hline
\end{tabular}

According to Table 3, we can conclude that the BEIR is an unbiased estimator for inflation only for the maturity of three months. For other maturities $-6,9,12,15,18,24,30$ and 36 months - when the BEIR bring relevant information about inflation, it does in a biased fashion.

\section{The Predictive Power of the BEIR}

We showed in the previous section that the BEIR bring relevant information about future inflation and that it may be considered an unbiased estimator of inflation for the maturity of three months. The natural extension of this result is exactly the secondary objective of this study: to determine the predictive power of the BEIR against other measures of inflation expectations, such as those extracted from Focus Survey Report and the VAR models of the Central Bank of Brazil.

The choice of inflation expectations extracted from the Focus Survey Report can be easily justified by its availability, since it is a free access publication, coverage, since it presents expectations from about 90 banks and non-financial businesses, and weekly publication, with the results of research conducted daily. Specifically for the purpose of our work, we considered the median expectation for the CPI, extracted from the Top5 institutions. The Top5s are the five best forecasting institutions among the participants of the Focus Survey Report. The choice of institutions in the medium-term rank rather than in the short-term rank is due to the forecast horizon considered for classification. The short-term 
rank considerers only one-month ahead forecasts, while the medium-term rank considers four-months ahead forecasts. This means that the institutions ranked in the medium-term Top5s have developed more robust models for longer time horizons.

The use of VAR models for the exercise proposed in this section can also be easily justified: the Central Bank of Brazil, as well as many of its international peers, uses VAR models for analysis and inflation-forecasting. In the case of the Brazilian monetary authority, VAR models were introduced to the analysis process after the implementation of the inflation-targeting regime, in June 1999. These models were reviewed over the past years, still constituting an important part of the decision-making process of the Monetary Policy Committee of the Central Bank of Brazil. In addition, VAR models impose few restrictions on the structure of the economy. We have to choose, basically, the variables to be used and the lags, all the rest is determined by the model. This is a desirable feature in the kind of exercise we proposed in this section, since it reduces the degree of subjectivity in the analysis.

The VAR models of the Central Bank of Brazil are divided, essentially, into two major groups: models with economic fundamentals and purely statistical models. Because they do not have economic restrictions - in the short or long-term - the purely statistical VAR models were not considered in our analysis. Among the models with economic fundamentals, only those on a monthly basis were considered, to allow comparison with the predictions of the BEIRs, and were estimated in a traditional, non-Bayesian, fashion. Therefore, we have estimated only three VAR models:

Table 4 - The VAR Models of the Central Bank of Brazil

\begin{tabular}{|c|c|c|c|}
\hline Model & Endogenous Variables & $\begin{array}{c}\text { Seasonally } \\
\text { Ajusted }\end{array}$ & Lags \\
\hline VAR I & Inflation, Exchange Rate and Real Interest Rate & Yes & 2 \\
\hline VAR II & Inflation, Exchange Rate, Nominal Interest Rate, Industrial Production and Monetary Aggregate & Yes & 6 \\
\hline VAR III & Inflation, Exchange Rate and Industrial Production & No & 1 \\
\hline
\end{tabular}


In the estimation of the VAR models, we used the CPI for inflation, unlike the Central Bank, that estimates market and monitored prices separately. The purpose of this amendment is to avoid introducing subjectivity in the analysis. In addition to the CPI, we used: the BRL/USD exchange rate (PTAX), end of period; the Selic rate (\% p.a.), as nominal interest rate; the inflation measured by the IGP-DI - a Brazilian general price index -, deducted from the Selic rate (\% p.a.) as real interest rate; industrial production seasonally adjusted; and, finally, M1 as the monetary aggregate. The exercise considered, as well, the average of forecasts from the VAR models. The comparison of the forecast errors for different maturities and models is presented in the following table:

Table 5 - Prediction Errors

\begin{tabular}{cccccccccc}
\hline & \multicolumn{9}{c}{ Mean Square Error (Square Root) } \\
\hline BEIR & $\mathbf{3}$ & $\mathbf{6}$ & $\mathbf{9}$ & $\mathbf{1 2}$ & $\mathbf{1 5}$ & $\mathbf{1 8}$ & $\mathbf{2 4}$ & $\mathbf{3 0}$ \\
VAR I & 2.18 & 1.93 & 1.66 & 1.51 & 1.54 & 1.55 & 1.50 & 1.50 \\
VAR II & 3.56 & 3.17 & 3.22 & 3.32 & 3.43 & 3.49 & 3.52 & 3.34 & 3.46 \\
VAR III & 4.08 & 3.93 & 3.32 & 2.96 & 2.88 & 2.91 & 3.08 & 3.42 & 3.54 \\
Average VARs & 2.85 & 2.97 & 2.96 & 3.02 & 3.19 & 3.34 & 3.37 & 3.44 & 3.51 \\
Top5s & 2.74 & 2.73 & 2.58 & 2.55 & 2.58 & 2.58 & 2.48 & 2.35 & 2.29 \\
& 1.97 & 1.44 & 1.26 & 1.23 & - & - & - & - & - \\
\hline
\end{tabular}

From Table 5, we conclude that the medium-term Top5s present the lowest inflation forecasting error. The predictions from the Top5s, for most of the sample, were performed only for 12 months ahead - because of a limitation in the expectation`s system of the Central Bank of Brazil -, limiting the comparison to the maturities of 15, 18, 24, 30 and 36 months. For these maturities, the BEIRs show greater accuracy than the VAR models. The best result for the VAR models was generated by the average of VAR I, VAR II and VAR III forecasts.

This is an important result, because it suggests that the BEIRs outperform the VAR models considered - individually or their average - in inflation forecasting, measured by the 
CPI, at all maturities. The medium-term Top5s have the smallest forecasting errors, outperforming the projections generated by both the BEIRs and VAR models. This result, to some extent, was expected, since the Top5s use this and other VAR models, as well as more complex models, such as structural VAR models, structural macroeconomic models, among others.

\section{Conclusion}

The present paper aimed to evaluate, for the Brazilian case, if break-even inflation rates bring relevant information about future inflation, without the restrictive assumptions commonly imposed by the literature. We proposed an innovative way to estimate this relationship, by the Kalman filter, to allow the risk premium and the explanatory power of the BEIR to vary over time.

Our estimations suggested that the BEIR carries information about future inflation. The coefficients associated with the BEIRs were positive and statistically significant for 3, 6 and 9 months. For 12 and 15 months, the coefficients associated with the BEIRs were not significant, while for 18, 24, 30 and 36 months, the coefficients, though significant, were negative. The risk premium, represented by the constant term, was estimated to be statistically different from zero and very close to this value for all maturities. The compensation for incurred risk was time-varying for some to maturities, such as $15,18,24$, 30 and 36 months.

We also evaluated if the BEIR could be considered an unbiased estimator for inflation. For this to be true, it was necessary that, jointly, the constant was equal to zero and the coefficient associated with the BEIR equal to unity. We performed a F-test to assess the validity of this hypothesis for each of the maturities, concluding that the BEIR may be considered an unbiased estimator for inflation only for the maturity of three months. 
Finally, we evaluated the predictive power of the BEIRs against other measures of inflation expectations, such as those extracted from VAR models used by the Central Bank of Brazil and estimates from the Focus Survey Report's Top5s. Our estimates indicated that the forecasts generated from the BEIRs showed higher accuracy than those obtained from the VAR models. These projections, however, underperformed those from the Top5s. 


\section{References}

ANG, A.; BEKAERT, G.; Wei, M. (2007). Do Macro Variables, Asset Markets Forecast Inflation Better? Journal of Monetary Economics, 54, 1163-1212.

BARR, D.G.; CAMPBELL, J.Y. (1997). Inflation, real interest rates, and the bond market: a study of UK nominal and index-linked government bond prices. Journal of Monetary Economics, Vol. 39(3), p. 361-383.

BERNANKE, B. (2004). What policy makers can learn from asset prices. < http://www.federalreserve.gov/boarddocs/speeches/2004/20040415/default.htm >.

BREEDON, F. (1995). Bond prices and market expectations of inflation. Bank of England, Quarterly Bulletin, May, p. 160-165.

BURASCHI, A.; JILTSOV, A. (2005). Inflation Risk Premia and the Expectation Hypothesis, Journal of Financial Economics, 75, 429-490.

CALDEIRA, J. F. (2011). Estimação da Estrutura a Termo da Curva de Juros no Brasil Através de Modelos Paramétricos e Não- Paramétricos. Análise Econômica.

CAMPBELL, J.Y.; SHILLER, R.J. (1996). A Scorecard for Indexed Debt. NBER Macroeconomics Annual, p. 155-197.

CHEN, R. R.; LIU, B.; CHENG, X. Inflation, Fisher Equation, and the Term Structure of Inflation Risk Premia: Theory and Evidence from TIPS. Journal of Empirical Finance (forthcoming).

D'AMICO, S.; KIM, D. H.; WEI, M. (2006). Tips from TIPS: the informational content of Treasury Inflation-Protected Security Prices, Working paper, Federal Reserve Board.

DEACON, M.; ANDREWS, P. (1996). The use and value of index-linked bonds. The Financier, Vol. 3.

DURBIN, J.; KOOPMAN, S. (2001). Time Series Analysis by State Space Methods. Oxford, New York.

EMMONS, W.R. (2000). The information content of Treasury Inflation-Indexed Securities. Federal Reserve Bank of St Louis, Review, November/December, p. 25-37.

EVANS, M. (1998). Real Rates, Expected Inflation, and Inflation Risk Premia. The Journal of Finance, 53(1), 187-218.

GARCIA, J. A.; VAN RIXTEL, A. (2007). Inflation-linked bonds from a central bank perspective. European Central Bank, Occasional Paper Series, N. 62.

GRISHCHENKO, O.; HUANG, J. (2010). Inflation Risk Premium: Evidence from the TIPS market. Working Paper, <http://ssrn.com/abstract=1364254>. 
HAUBRICH, J.; PENNACCHI, G.; RITCHKEN, P. (2008). Estimating Real and Nominal Term Structures using Treasury Yields, Inflation Forecasts, and Inflation Swap Rates, Working Paper, Federal Reserve Bank of Cleveland, University of Illinois at UrbanaChampaign and Case Western Reserve University.

HETZEL, R.L. (1992). Indexed bonds as an aid to monetary policy. Federal Reserve Bank of Richmond, Economic Review, January/February, p. 13-23.

HORDAHL, P.; Tristani, O.; D. Vestin, D. (2008). The yield curve and macroeconomic dynamics. Economic Journal, 118(533), 1937-70.

JARROW, R.; Yildirim, Y. (2003). Pricing Treasury Inflation Protected Securities and Relative Derivatives using HJM Model, Journal of Financial and Quantitative Analysis, 38(2), 337-358.

KIM, C.J.; NELSON, C.R. (1999). State-Space Models with Regime Switching. Cambridge, Massachusetts: MIT Press.

KITAMURA, Y. (1997). Indexed bonds and monetary policy: the real interest rate and the expected rate of inflation. Bank of Japan, Monetary and Economic Studies, Vol. 15(1), p. 125.

SVENSSON, L. E. O. (1994). Estimating and Interpreting Forward Interest Rates: Sweden 1992-1994. IMF Working Papers 94/114. International Monetary Fund.

VICENTE, J.V.M.; GUILLEN, O.T.C. (2010). Do inflation-linked bonds contain information about future inflation? Central Bank of Brazil, Working Paper Series, N. 214. 